\title{
Postural and respiratory function of the abdominal muscles: A pilot study to measure abdominal wall activity using belt sensors
}

\author{
Jakub Novak $^{\mathrm{a}, *}$, Andrew Busch ${ }^{\mathrm{b}}$, Pavel Kolar ${ }^{\mathrm{a}}$ and Alena Kobesova ${ }^{\mathrm{a}}$ \\ ${ }^{a}$ Department of Rehabilitation and Sports Medicine, Second Faculty of Medicine, Charles University and University \\ Hospital Motol, Prague, Czech Republic \\ ${ }^{\mathrm{b}}$ Department of Health and Human Kinetics, Ohio Wesleyan University, Delaware, OH, USA
}

Received 15 August 2020

Accepted 1 October 2020

\begin{abstract}
.
BACKGROUND: The abdominal muscles play an important respiratory and stabilization role, and in coordination with other muscles regulate intra-abdominal pressure (IAP) to stabilize the spine.

OBJECTIVE: To examine a new, non-invasive method to measure activation of the abdominal wall and compare changes in muscle activation during respiration while breathing under a load, and during instructed breathing.

METHODS: Thirty-five healthy individuals completed this observational crossover study. Two capacitive force sensors registered the abdominal wall force during resting breathing stereotype, instructed breathing stereotype and under a load.

RESULTS: Mean abdominal wall force increased significantly on both sensors when holding the load compared to resting breathing (Upper Sensor: $P<0.0005, d=-0.46$, Lower Sensor: $P<0.0005, d=-0.56$ ). The pressure on both sensors also significantly increased during instructed breathing compared to resting breathing (US: $P<0.0005, d=-0.76$, LS: $P<0.0005$, $d=-0.78$ ).

CONCLUSIONS: The use of capacitive force-sensors represent a new, non-invasive method to measure abdominal wall activity. Clinically, belts with capacitive force sensors can be used as a feedback tool to train abdominal wall activation.
\end{abstract}

Keywords: Spinal stabilization, respiration stereotype, intra-abdominal pressure (IAP), capacitive force sensors

\section{Introduction}

Over the past two decades, numerous authors have investigated lumbar spine stabilization [1,2], motor control of the trunk muscles $[3,4]$, and the regulation of intra-abdominal pressure [5,6]. Balanced coordination between the diaphragm, pelvic floor muscles, and abdominal wall musculature is critical for IAP regulation

\footnotetext{
${ }^{*}$ Corresponding author: Jakub Novak, Department of Rehabilitation and Sports Medicine, Second Faculty of Medicine, Charles University and University Hospital Motol, V Uvalu 84, Prague 5, 150 06, Czech Republic. Tel.: + 42022443 9264; Fax: +420 22443 9220; E-mail:kuba-novak@seznam.cz.
}

which forms an important spinal stabilization mechanism [7-9]. The pelvis and lumbar spine are reflexively stabilized before the limb movements start [10-12]. This feed forward stabilization mechanism is secured by the trunk muscles. The diaphragm works in conjunction with the pelvic floor and deep intrinsic muscles of the spine and the transversus abdominis to create stiffness and minimize other intrinsic and extrinsic stressors to the spine during motion [13]. Biomechanical studies confirm that pressurization of the abdomen increases lumbar spinal stability, but the degree of spinal stability is not substantially influenced by selective activation of certain abdominal muscles [7]. Forced activity of transversus or obliques may cause reductions in the ac- 
tivation of other abdominal muscles and even produce decreased lumbar stability [7].

Appropriate functional tone and conditioning the abdominal wall to work as one harmonic unit with the pelvic floor musculature is a critical stabilization component that optimizes the "push back" of the viscera up into the diaphragm, helping expansion of the lower rib cage for respiration [13]. According to Wallden a deconditioned or inhibited abdominal wall causes visceroptosis, reducing the pressure the viscera exerts into the diaphragm to open the lower rib cage for inhalation, and driving the diaphragm back upward during full exhalation [13]. The postural-respiratory function of the trunk muscles is inseparable. With heavy load lifting, trunk muscle activation in the abdominal and thoracic cavities act like a rigged-walled cylinder, providing increased spinal stability [14]. The regulation of IAP within the abdominal wall in coordination with diaphragm and pelvic floor muscles significantly contributes to spinal stabilization and protects the spine during loading $[15,16]$. Perhaps not only the amount of the abdominal wall activation but also the type of contraction, i.e. eccentric vs. concentric plays a role in spinal stabilization. With postural activites, the diaphragm descends caudally to pressurize intra-abdominal contents [17]. The pelvic floor must support the viscera from bellow. Since the viscera are non-compressible, one can assume that the abdominal wall must react to diaphragmatic descend by eccentric contraction. Insufficient distension or exccesive initial concentric contraction of the abdominals preventing diaphragmatic descend during postural activites may compromise the whole stabilization mechanism. Poor coordination of postural muscles and insufficient stabilizing function of deep back muscles, diaphragm, abdominal and pelvic floor muscles may result in spinal disorders associated with back pain, such as deformation spondyloarthrosis, intervertebral disc protrusion or spondylolisthesis $[18,19]$.

Various measurement procedures have been used to investigate the postural-respiratory function of the abdominal muscles and related IAP changes. Esohpageal, gastric [20], intravesical [21], anal [22,23], and vaginal [24] probes can measure IAP, yet these methods are often time consuming and uncomfortable for patients. Electromyography [23] and ultrasound [25] assessments have also been used to analyze activity of the abdominal muscles, but these methods can be burdensome, technically demanding to perform while the former poses serious difficulty regarding its reproducibility and hence interpretation.
Table 1

Descriptive statistics of the sample

\begin{tabular}{lllll}
\hline & Age (years) & Height $(\mathrm{cm})$ & Weight $(\mathrm{kg})$ & BMI \\
\hline Mean & 21.26 & 170.51 & 63.17 & 24.07 \\
SD & 1.62 & 6.49 & 7.94 & 3.02 \\
Min & 19 & 160 & 47 & 17.27 \\
Max & 25 & 185 & 80 & 27.62 \\
\hline
\end{tabular}

This paper presents a new, non-invasive method to measure force of the abdominal wall using capacitive force sensors, which may help medical practicioners provide quick, visual information to patients regarding their postural-respiratory function.

Therefore, the purpose of this research was to compare how activation of abdominal muscles changes during respiration while breathing under a load and when being instructed to modify breathing stereotype in a healthy population.

\section{Methods}

\subsection{Participants}

This study included 35 healthy individuals, 8 males and 27 females, aged 19-25 years. Table 1 shows descriptive statistics of the sample. The participants have not experienced acute or chronic musculoskeletal pain, reported no pain during the measurements, never suffered from any serious trunk pathologies, never undergone any trunk operations and have never received any therapy or training focusing on intra-abdominal pressure activation or abdominal wall expansion. Individuals with body mass index (BMI) over 30 were excluded from the study. Written informed consent was obtained from each participant, and this study was approved by an Institutional Ethics Committee (Ethics Committee of the University Hospital Motol and $2^{\text {nd }}$ Faculty of Medicine, Charles University in Prague. No. 1263.1.15/19; approval date: November 6, 2019). The study conforms with The Code of Ethics of the World Medical Association.

\subsection{Instruments}

For the noninvasive examination of abdominal muscles function, a unique device called Ohm Belt (Nilus Medical LLC, 2019 (c) OHMBELT, Redwood City, CA, USA). A research version of the device was designed by the manufacturer for the trial purposes, which differs from the commercial version in that two sensors recorded data simultaneously with a software app to 

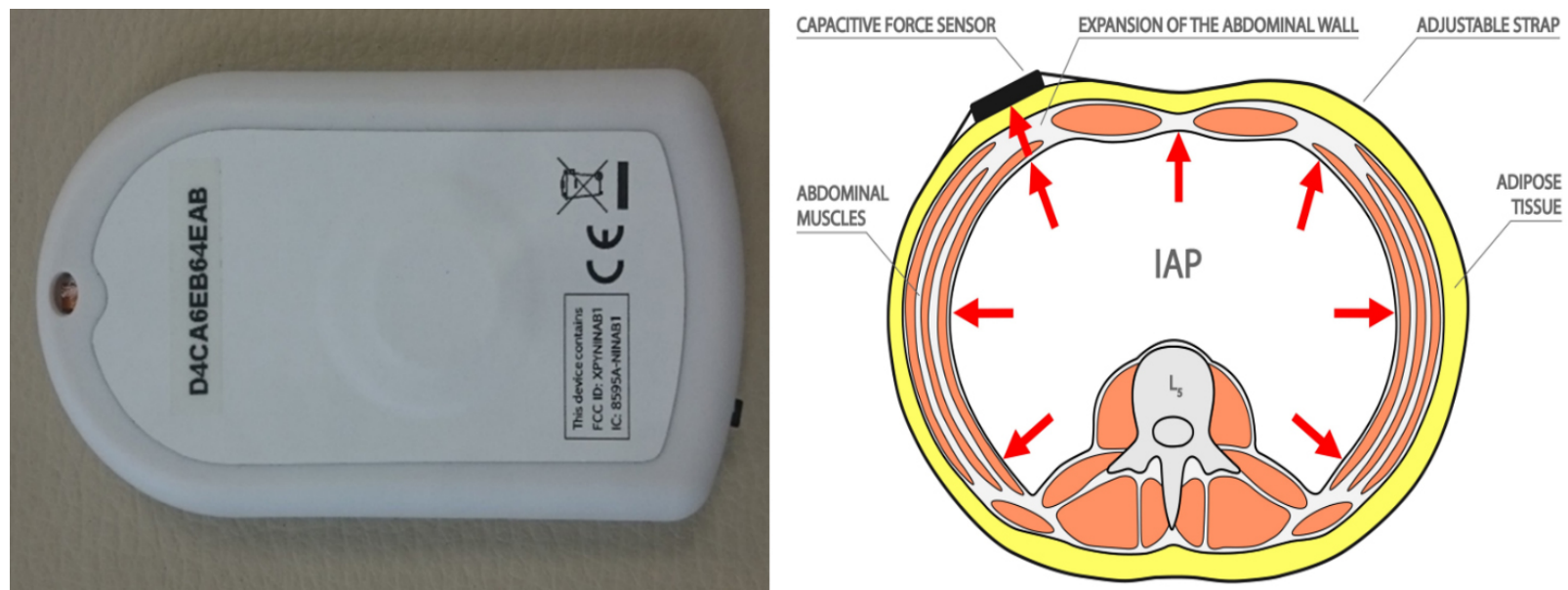

Fig. 1. Close-up picture of the capacitive force sensor and a scheme of abdominal cavity with expansion of the abdominal wall and attached sensor.

display and record both sensor force data. It consists of two capacitive force sensors of $15 \mathrm{~mm}$ diameter, $0.35 \mathrm{~mm}$ thickness, full scale range $0.45 \mathrm{~kg}$, minimal detectable force $0.9 \mathrm{~g}$ (Fig. 1), attached to the abdominal wall by adjustable straps. The device utilizes forcesensing capacitor type of sensor, which consists of a material whose capacitance changes when a force is applied. Such sensors are also known as "force-sensitive capacitors" and reported to be used in other medical research projects [26-28]. This sensor is an example of parallel plate capacitator (see Fig. 1). For small deflections, there is a linear relationship between applied force and change in capacitance. Force sensor, facing the subject's skin, is pressed against the abdominal wall by the adjustable firm strap (see Fig. 1). Abdominal wall expansion and retraction is recorded by the sensor as a force (The "abdominal wall force"). The sensors register the force exerted by the abdominal wall during respiration and various postural tasks. The abdominal wall force is measured in grams over a period of time, where $1 \mathrm{~g}=0.01 \mathrm{~N}$. The gram scale was selected to provide users with feedback in integer rather than decimal values. Abdominal wall force was measured by the sensor and recorded in grams over a period of time. The force displayed on the graph scale in grams is, therefore, technically in the gram-force unit (gf). The dualchannel pressure sensor consists of two sensors which monitor simultaneously the instantaneous muscle force at two different locations. Both the amount of the force and its dynamics over time can be analyzed. The sensors are also equipped with accelerometers to capture any kyphotic trunk synkinesis, i.e. substitutive trunk movement replacing abdominal muscle activation. A built-in tensometric transducer converts the force to the digital signal that is transmitted wirelessly via bluetooth to the computer where the software graphically displays the results. The program records any time sequences with the numerical values being automatically exported into MS Excel. Immediate data analysis, graphical imaging and data saving is available.

\subsection{Assessments}

The assessments of all participants were performed under the same conditions (daytime, assessment room, temperature), and by the same examiner. Each participant was in an upright seated position, with hips and knees flexed at $90^{\circ}$, and both feet supported on the floor (Fig. 2). First, a pilot study was performed on 20 healthy individuals to measure abdominal wall activity in various postural and breathing situations, using different ways of fixation and placement of the sensors to achieve maximal measurement accuracy and sensitivity. By repeated measurements, it was determined that fixation with firm but flexible belts under the pressure of of 120 grams $\pm 10 \mathrm{~g}$ for the upper sensor (US) and 140 grams $\pm 10 \mathrm{~g}$ for the lower sensor (LS) allows for sufficiently accurate measurements while not limiting trunk and abdominal wall movements. Before each measurement, boths sensors were calibrated to a baseline of zero and positioned on each participant using palpation by a skilled manual therapist. The US was placed on the superior trigonum lumbale, bellow the floating ribs, and the LS was placed above the groin at the intersection of the mammilar and bispinal connecting line. Sensors were randomly placed on the left or right side of the body. The sensors were fixed during tidal expirium.

The participants were instructed to maintain the upright sitting position throughout the course of the whole 

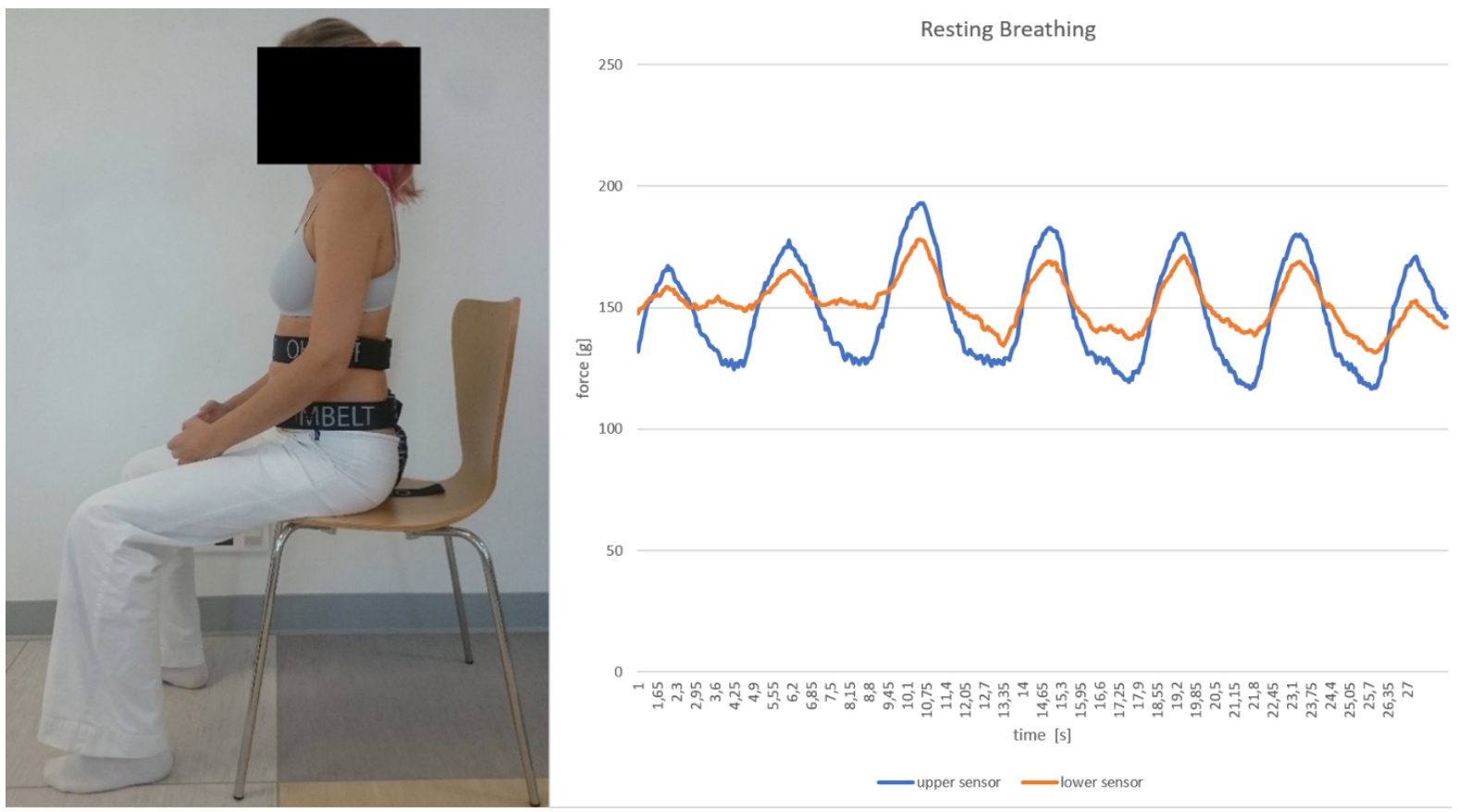

Fig. 2. Resting breathing assessment. Force sensors are placed under the belts.

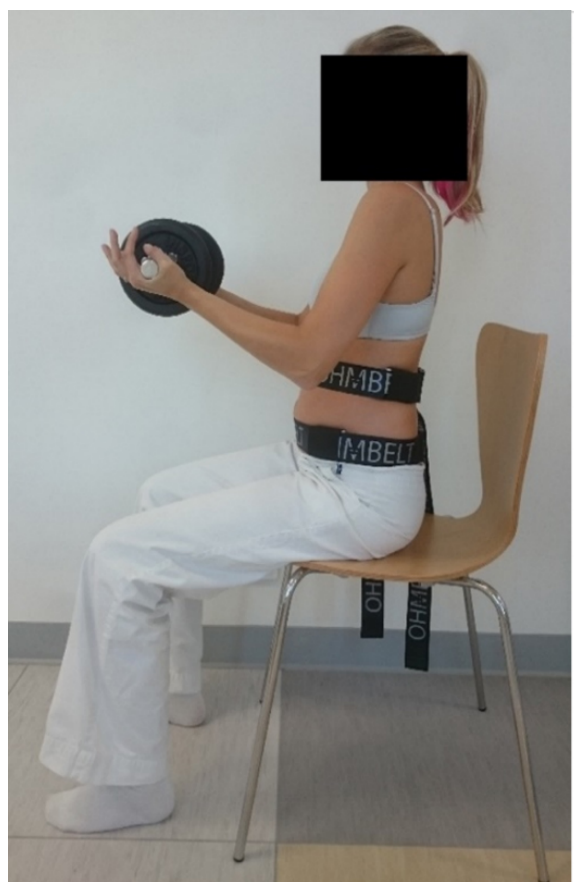

Load Breathing

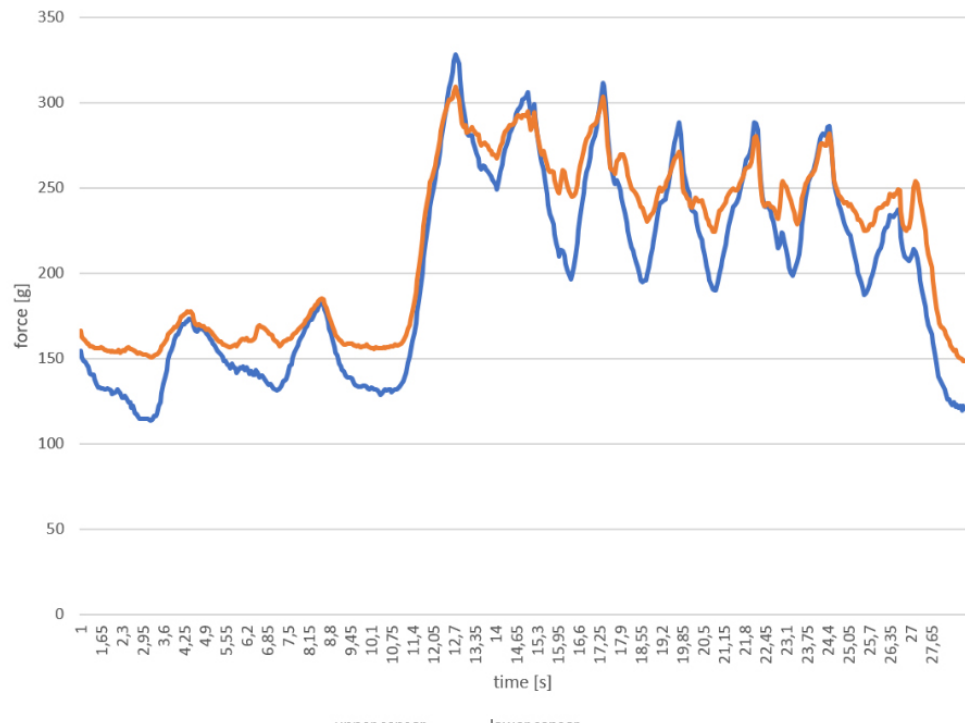

Fig. 3. Load breathing assessment. At the 10th second the weight was put into participant's hands (note the steep increase of both lines in the graph).

assessment, avoiding increased spinal kyphosis or lordosis. In all individuals, the abdominal wall activity was recorded for a total period of 15-20 seconds for each scenario. Abdominal activity fluctuated when each participant began to hold the load, and upon releasing the load, so only 10-second intervals of stable activation 

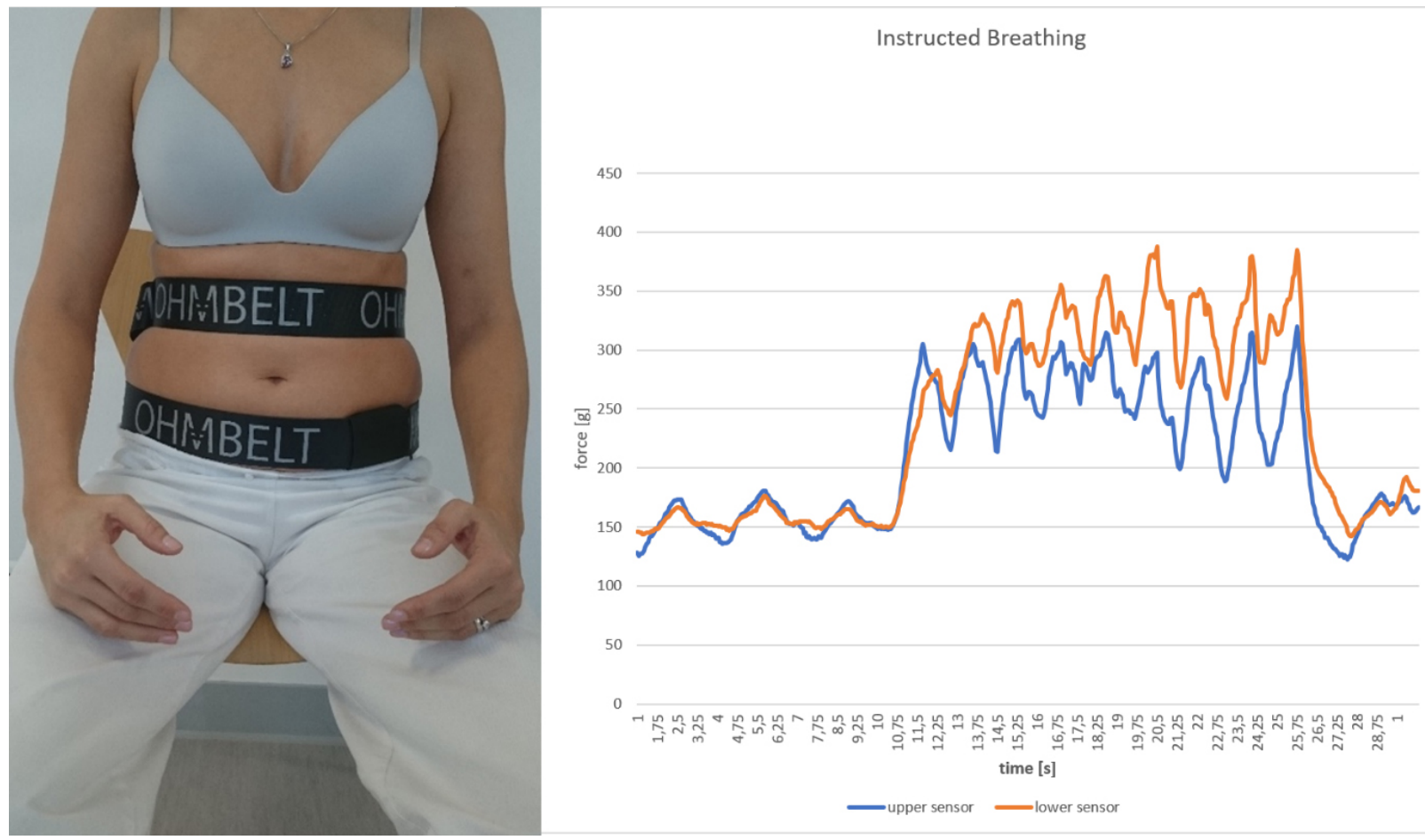

Fig. 4. Instructed breathing assessment. First, the individual breathes normally, then at the 10th second he/she was instructed to direct the breath towards both sensors (steep increase of both lines in the graph).

were used for the mean statistical analysis calculated separately for each sensor. First, the natural stereotype of resting breathing (RB) was monitored (scenario 1, Fig. 2). Then, the participants were breathing naturally when sitting upright and holding a load corresponding to $20 \%$ of their body weight with elbows flexed at $90^{\circ}$. This was scenario 2, i.e. load breathing (LB), (Fig. 3). And finally, instructed breathing (IB) was monitored (Fig. 4). The participants were instructed to voluntarily and maximally expand their abdominal wall, directing inspiration towards the sensors and maintaining maximum pressure contact with the sensors during expiration.

\subsection{Statistical analysis}

Descriptive statistics were calculated for each measure. Paired-samples $t$ tests were used to compare abdominal wall pressure in both sensors during resting breathing with the interventions, and also to compare inter-sensor differences between measurements during each condition. Cohen's d effect sizes were calculated for the differences between breathing conditions as the difference between groups divided by the pooled standard deviation. Effect sizes were interpreted as very small $(<0.2)$, small $(0.2-0.5)$, medium $(0.5-0.8)$, or large $(>0.8)$. Power analysis, using $G^{*}$ Power 3.1, indicated an $80 \%$ chance of detecting a medium effect size of 0.5 in 34 subjects with statistical significance determined $a$ priori at $p<0.05$ (two-tailed). Data analyses were conducted using the Statistical Package for the Social Sciences (SPSS version 26.0 for Mac; IMB Corp, Armonk, NY).

\section{Results}

Thirty-five participants completed the study. Table 2 presents the amount of force of the abdominal wall (g) during resting breathing, loaded breathing and instructed breathing $(n=35)$ for both sensors. The paired sample $t$-test indicated that the mean abdominal wall force increased significantly on both sensors when holding the load compared to the resting breathing ( $P$ for both sensors $<0.0005)$. The force on both sensors also significantly increased during instructed breathing compared to the resting breathing ( $P$ for both sensors $<$ $0.0005)$. Figure 5 depicts the results.

The inter-sensor difference was also compared for all three measured scenarios (resting, under load, and 
Table 2

External force changes of the abdominal wall (g) during loaded breathing and instructed breathing $(n=35)$

\begin{tabular}{clccrrrr}
\hline \multirow{2}{*}{ Measure } & $\begin{array}{c}\text { Breathing } \\
\text { intervention }\end{array}$ & $\begin{array}{c}\text { Resting } \\
\text { mean }(\mathrm{SD})\end{array}$ & $\begin{array}{c}10 \text { second } \\
\text { mean }(\mathrm{SD})\end{array}$ & $\begin{array}{c}\text { Mean } \\
\text { difference }(\mathrm{SD})\end{array}$ & $\begin{array}{c}95 \% \text { CI of } \\
\text { difference }\end{array}$ & $\begin{array}{c}\text { Effect size } \\
P \text { Value }\end{array}$ \\
\hline \multirow{2}{*}{ Upper sensor } & Loaded & $123.75(21.95)^{* *}$ & $165.07(39.44)^{* *}$ & $-41.32(38.00)$ & $(-54.38,-28.27)$ & -0.46 & $<0.0005^{*}$ \\
& Instructed & & $228.31(64.96)$ & $-104.56(60.64)$ & $(-125.39,-83.73)$ & -0.76 & $<0.0005^{*}$ \\
Lower sensor & Loaded & $141.98(18.19)^{* *}$ & $191.74(40.51)^{* *}$ & $-49.76(39.79)$ & $(63.43,-36.09)$ & -0.56 & $<0.0005^{*}$ \\
& Instructed & & $249.02(66.09)$ & $-107.04(63.79)$ & $(-128.95,-85.13)$ & -0.78 & $<0.0005^{*}$ \\
\hline
\end{tabular}

* Statistical difference observed between conditions $(P<0.05) .{ }^{* *}$ Statistical difference observed between sensors $(P<0.05)$. Note: Values are (g). Effect size $=$ calculated Cohen's d.

Force Changes in Upper and Lower Sensors (g)

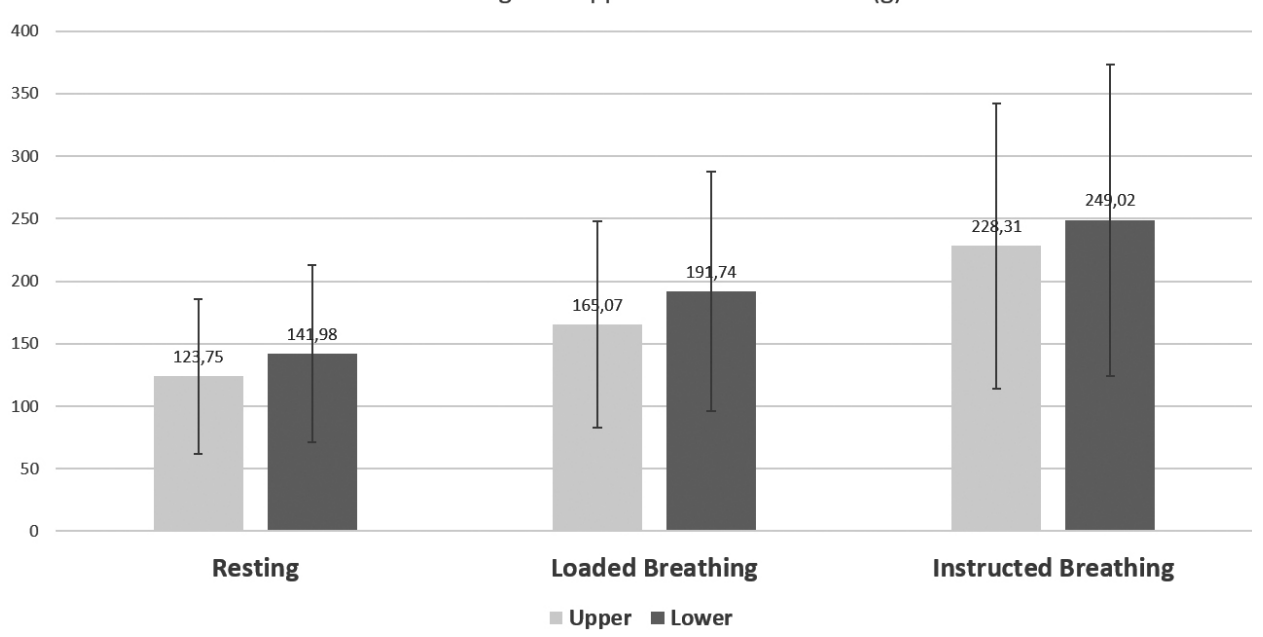

Fig. 5. Statistical comparison of forces on both sensors at resting, loaded, and instructed breathing.

instructed breathing). There was a significantly greater force on the lower sensor in the resting condition ( $p<$ $0.0005)$, and in the loaded condition $(p=0.001)$, but not in the instructed breathing condition $(p=0.068)$. Figure 5 depicts the inter-sensor difference.

\section{Discussion}

This study presents a new method to analyze activity of the abdominal wall using capacitive force sensors. Originally, the sensors were produced by a medical technology developer to offer feedback to LBP clients training optimal breathing stereotype. Research suggests patients with low back pain (LBP) often demonstrate sub-optimal respiratory parameters $[29,30]$ and confirms a positive effect of breathing exercises on LBP $[31,32]$. Clinically, insufficient activation of the latero-dorsal sections of the abdominal wall (i.e. in superior trigonum lumbale and over the lower abdominal wall just above the groin) are common findings in LBP patients $[33,34]$. Therefore, the sensors were placed in these locations because they are usually the most diffi- cult to voluntarily activate in an eccentric manner. Also, a similar placement of abdominal wall pressure measuring device was previously used in studies published by other authors $[35,36]$. Synergy between the diaphragm, pelvic floor, abdominal wall and spinal extensors is necessary to stabilize the spine. During stabilization, the concentric activity of the diaphragm is followed by the eccentric activity of the entire abdominal wall. This synergy increases intra-abdominal pressure (IAP) that stabilizes the lumbar spine anteriorly, balancing with the spinal extensors that secure stabilization posteriorly [37]. Based on existing literature [6,38,39], we assume the pressure monitored by the sensors is not only activity exerted by the abdominal muscles but mainly the IAP resulting from the complex coordination of all core stabilizers.

This study confirms that pressure exerted by the abdominal wall increases with increased postural load, because the activity measured on both sensors during $\mathrm{LB}$ was significantly higher comparing to RB. This further supports electromyography (EMG) studies reporting increased abdominal muscle activity during posturally challenging situations [40] and when lifting the 
load [41], as well as studies confirming IAP elevation during postural tasks and load lifting [9,37]. Withinlimb movements, and coactivation of the diaphragm with abdominal muscles cause an increase in IAP [37]. This is a very important stabilizing mechanism because IAP elevation increases stiffness of the lumbar spine $[9,42]$. Applying simple and non-invasive capacitive force sensors, we obtained similar results reported by other complicated and sophistic methods such as multichannel EMG analysis or IAP gastric, esophageal or intra-annal pressure measurements $[23,25]$. Furthermore, we statistically confirmed that healthy, young individuals are able to voluntarily activate the coordination between the diaphragm, abdominal muscles and pelvic floor muscles, because the force on both sensors significantly increased during IB comparing to RB. This again, supports other studies showing how breath control and abdominal muscle control influences IAP $[23,43]$. We purposefully performed the IB test at the end of the assessment to exclude any learning process. First resting and loaded breathing spontaneous uncorrected stereotypes were analyzed and only then were instructions given on how to breath. The individuals were cued to direct the breath towards both sensors. The goal was to measure the amount of voluntary pressure exerted on sensors during maximum inhalation directed to the sensors. The second goal was to confirm it is actually possible to teach participants how to voluntarily activate the abdominal wall sections under the sensors. This may be of potential benefit in the treatment and training of LBP individuals who frequently demonstrate an inability to activate such areas of the abdominal wall both during breathing and postural tasks $[33,34]$. Further studies are needed to confirm.

Interestingly, this study showed that postural activation when holding a load corresponding to $20 \%$ body weight (LB scenario) requires, on average, about twothirds of the maximum muscle coactivation generated by the conscious maximum force of the abdominal wall against the sensors (IB scenario). Similarly, Essendrop et al. reported IAP increased from 0 to $40 \%$ when holding a load of $15 \%$ body weight [42]. However, it remains questionable what amount of activation would occur when holding a heavier gload, which is common during activities of daily living or sport.

A similar measurement of the abdominal wall expansion was done by Malatova et al. using an electromechanical dynamometer [44-46]. The authors describe how muscular dynamometers can be used to evaluate activation of the whole deep stabilizing spine system (DSSS) because abdominal muscles comprise the
DSSS, arguing that the DSSS works as one functional unit and dysfunction of just one DSSS muscle is related to total dysfunction of the whole muscle system [44]. Malatova also used the dynamometer to analyze the effect of a six-week intervention program focusing on optimal body posture and DSSS training [46]. Comparing to Malatova's rather big muscular dynamometer, these sensors are smaller in size and transmit signals to a PC via Bluetooth, allowing practicioners to analyze more dynamic situations including all types of exercises. Also, unlike Malatova's measuring device, the sensors used in this study are commercially available (Nilus Medical LLC, 2019 (C) OHMBELT).

In a study on 45 asymptomatic women, Malatova identified more excessive activation of the upper sections of the abdominal wall compared to lower sections, questioning if this is a natural or pathological stereotype [44]. Our study demonstrated significantly greater pressure on the lower sensor in the RB condition $(p<$ $0.0005)$, and in the LB condition $(p=0.001)$, for the IB condition the activity on the lower sensor was also greater but not statistically significant $(p=0.068)$. This topic deserves more research attention. It is necessary to determine how much difference can be considered physiological and what is already abnormal. Postural responses of the abdominal muscles differ between body positions, the recruitment and contribution of abdominal muscle regions to stabilize the trunk varies, and regional differentiation in abdominal muscle activity may be a natural stabilization mechanism [47].

It has been shown that selective activation of the individual abdominal muscles does not significantly increase spinal stability [7]. Spinal stabilization training should therefore focus on global coordination of all muscles involved in stabilization function and IAP regulation rather then addressing individual abdominal muscles. Abdominal wall pressure changes were previously monitored by other authors $[35,36]$ reporting inverse significant correlations between ability to activate the abdominal wall and pain severity in LBP population [36]. Esophageal, gastric, anal or vaginal sensory measuring IAP $[9,48]$ as well as EMG $[3,48]$ or ultrasound [49] examination procedures help to analyze activity of the abdominal wall muscles. These techniques inform us about muscle activity but do not distinguish eccentric from concentric types of contraction and as a result do not provide information about an individual's ability to expand their abdominal wall. Abdominal wall expansion can be assessed clinically by palpation $[33,34]$, but clinical abdominal assessment showed poor sensitivity and accuracy for IAP pressure 
changes [50]. Trunk and abdominal circumference measurements can be highly influenced by the adipose tissues, therefore may also be unreliable [51]. The proposed method using capacitive force sensors may become a new, simple and non-invasive method to measure abdominal wall force. Future studies are needed to determine its sensitivity, accuracy and correlation with direct IAP measurements.

This study has some limitations. Based on available research $[6,38,39]$ we assume the detected amount of abdominal wall force is related to IAP changes, but IAP was not directly measured in this study. Future research correlating IAP with abdominal wall force would be useful in strengthening the findings of this study. This method of IAP detection may lose sensitivity in very obese individuals. The exact placement of the sensors would be less consistent due to difficult palpation of the anatomical landmarks, and the pressure measurement accuracy limited by a sick adipose tissue. Obese clients with BMI > 30 were excluded. Additionally, the sensor's fixation may alter the measurements. Too loose fixation of the belts reduces the sensitivity of the sensors, whereas too tight of fixation limits the ability to expand the abdominal wall, compromising the measured function. Placement of each sensor was unilaterally randomized, however in future studies we plan to use four sensors placed on both sides of the trunk to distinguish bilateral differences. Finally, this pilot study was performed on asymptomatic individuals, which does not mean all measured subjects performed with optimal muscular co-activation. In future studies, a larger number of individuals should be involved, comparing asymptomatic subjects with those suffering from LBP or other types of musculoskeletal problems.

\section{Conclusions}

This study presenst a new non-invasive method to measure abdominal wall force using belts with capacitive force sensors. The activity of the abdominal wall muscles significantly increases when lifting a load and with a purposeful activation during instructed breathing stereotype comparing to resting breathing. Future studies need to confirm if abdominal wall activation measured by the sensors correlates with direct IAP measurements.

\section{Acknowledgments}

We would like to thank Dr. Miroslav Navratil for providing us with the sensors.

\section{Author contributions}

CONCEPTION: Jakub Novak, Pavel Kolar and Alena Kobesova. PERFORMANCE OF WORK: Jakub Novak. INTERPRETATION OR ANALYSIS OF DATA: Jakub Novak and Andrew Busch.

PREPARATION OF THE MANUSCRIPT: Jakub Novak, Andrew Busch and Alena Kobesova. SUPERVISION: Alena Kobesova.

\section{Ethical considerations}

Written informed consent was obtained from each participant, and this study was approved by the Ethics Committee of the University Hospital Motol and $2^{\text {nd }}$ Faculty of Medicine, Charles University in Prague. No. 1263.1.15/19; approval date: November 6, 2019. The study conforms with The Code of Ethics of the World Medical Association.

\section{Conflict of interest}

There are no conflicts of interest to disclose.

\section{Funding}

This study was supported by The Charles University Grant Agency (GAUK No. 340220), and by Institutional research program Progres Q41.

\section{References}

[1] Cresswell AG, Oddsson L, Thorstensson A. The influence of sudden perturbations on trunk muscle activity and intraabdominal pressure while standing. Exp Brain Res. 1994; 98(2): 336-41.

[2] du Rose A. Have studies that measure lumbar kinematics and muscle activity concurrently during sagittal bending improved understanding of spinal stability and sub-system interactions? A systematic review. Healthcare (Basel). 2018 Sep 8; 6(3).

[3] Hodges PW, Moseley GL, Gabrielsson A, Gandevia SC. Experimental muscle pain changes feedforward postural responses of the trunk muscles. Exp Brain Res. 2003 Jul; 151(2): 262-71.

[4] Hirata RP, Salomoni SE, Christensen SW, Graven-Nielsen T. Reorganised motor control strategies of trunk muscles due to acute low back pain. Hum Mov Sci. 2015 Jun; 41: 282-94.

[5] Hodges PW, Cresswell AG, Daggfeldt K, Thorstensson A. In vivo measurement of the effect of intra-abdominal pressure on the human spine. J Biomech. 2001 Mar; 34(3): 347-53. 
[6] Cholewicki J, Juluru K, McGill SM. Intra-abdominal pressure mechanism for stabilizing the lumbar spine. Journal of Biomechanics. 1999 Jan 1; 32(1): 13-7.

[7] Stokes IAF, Gardner-Morse MG, Henry SM. Abdominal muscle activation increases lumbar spinal stability: analysis of contributions of different muscle groups. Clin Biomech (Bristol, Avon). 2011 Oct; 26(8): 797-803.

[8] Neumann P, Gill V. Pelvic floor and abdominal muscle interaction: EMG activity and intra-abdominal pressure. Int Urogynecol J Pelvic Floor Dysfunct. 2002; 13(2): 125-32.

[9] Hodges PW, Eriksson AEM, Shirley D, Gandevia SC. Intraabdominal pressure increases stiffness of the lumbar spine. J Biomech. 2005 Sep; 38(9): 1873-80.

[10] Aruin AS, Latash ML. Directional specificity of postural muscles in feed-forward postural reactions during fast voluntary arm movements. Exp Brain Res. 1995; 103(2): 323-32.

[11] Hodges PW, Richardson CA. Relationship between limb movement speed and associated contraction of the trunk muscles. Ergonomics. 1997 Nov; 40(11): 1220-30.

[12] Hodges PW, Cresswell AG, Daggfeldt K, Thorstensson A. Three dimensional preparatory trunk motion precedes asymmetrical upper limb movement. Gait Posture. 2000 Apr; 11(2): 92-101.

[13] Wallden M. The diaphragm - more than an inspired design. Journal of Bodywork and Movement Therapies. 2017 Apr; 21(2): 342-9.

[14] Goldish GD, Quast JE, Blow JJ, Kuskowski MA. Postural effects on intra-abdominal pressure during valsalva maneuver. Archives of Physical Medicine and Rehabilitation. 1994 Mar; 75(3): 324-7.

[15] Stokes IAF, Gardner-Morse MG, Henry SM. Intra-abdominal pressure and abdominal wall muscular function: spinal unloading mechanism. Clinical Biomechanics. 2010 Nov 1; 25(9): 859-66.

[16] Cholewicki J, Juluru K, Radebold A, Panjabi MM, McGill SM. Lumbar spine stability can be augmented with an abdominal belt and/or increased intra-abdominal pressure. Eur Spine J. 1999; 8(5): 388-95.

[17] Kolar P, Sulc J, Kyncl M, Sanda J, Neuwirth J, Bokarius AV et al. Stabilizing function of the diaphragm: dynamic MRI and synchronized spirometric assessment. J Appl Physiol. 2010 Oct; 109(4): 1064-71.

[18] Hodges PW, Richardson CA. Altered trunk muscle recruitment in people with low back pain with upper limb movement at different speeds. Arch Phys Med Rehabil. 1999 Sep; 80(9): 1005-12.

[19] Kalpakcioglu B, Altinbilek T, Senel K. Determination of spondylolisthesis in low back pain by clinical evaluation. J Back Musculoskelet Rehabil. 2009; 22(1): 27-32.

[20] Sugrue M, Buist MD, Lee A, Sanchez DJ, Hillman KM. Intraabdominal pressure measurement using a modified nasogastric tube: description and validation of a new technique. Intensive Care Med. 1994 Nov; 20(8): 588-90.

[21] Sugrue M, De Waele JJ, De Keulenaer BL, Roberts DJ, Malbrain MLNG. A user's guide to intra-abdominal pressure measurement. Anaesthesiol Intensive Ther. 2015; 47(3): 241-51.

[22] Kawabata M, Shima N, Hamada H, Nakamura I, Nishizono H. Changes in intra-abdominal pressure and spontaneous breath volume by magnitude of lifting effort: highly trained athletes versus healthy men. Eur J Appl Physiol. 2010 May; 109(2): 279-86.

[23] Tayashiki K, Takai Y, Maeo S, Kanehisa H. Intra-abdominal pressure and trunk muscular activities during abdominal bracing and hollowing. Int J Sports Med. 2016 Feb; 37(2): 134-43.
[24] Guaderrama NM, Nager CW, Liu J, Pretorius DH, Mittal RK The vaginal pressure profile. Neurourol Urodyn. 2005; 24(3): 243-7.

[25] Cresswell AG, Grundstrm̈ H, Thorstensson A. Observations on intra-abdominal pressure and patterns of abdominal intramuscular activity in man. Acta Physiol Scand. 1992 Apr; 144(4): 409-18.

[26] Egorov V, Sarvazyan AP. Mechanical imaging of the breast. IEEE Trans Med Imaging. 2008 Sep; 27(9): 1275-87.

[27] Salpavaara TJ, Verho JA, Lekkala JO, Halttunen JE. Embedded capacitive sensor system for hip surgery rehabilitation: Online measurements and long-term stability. In: 2008 30th Annual International Conference of the IEEE Engineering in Medicine and Biology Society. 2008. pp. 935-8.

[28] Ahmad HN, Barbosa TM. The effects of backpack carriage on gait kinematics and kinetics of schoolchildren. Sci Rep. 2019 04; 9(1): 3364.

[29] Shah SG, Choezom T, Prabu Raja G. Comparison of respiratory parameters in participants with and without chronic low back pain. J Bodyw Mov Ther. 2019 Oct; 23(4): 894-900.

[30] Calvo-Lobo C, Almazán-Polo J, Becerro-de-Bengoa-Vallejo R, Losa-Iglesias ME, Palomo-López P, Rodríguez-Sanz D, et al. Ultrasonography comparison of diaphragm thickness and excursion between athletes with and without lumbopelvic pain. Phys Ther Sport. 2019 May; 37: 128-37.

[31] Anderson BE, Bliven KCH. The use of breathing exercises in the treatment of chronic, nonspecific low back pain. J Sport Rehabil. 2017 Sep; 26(5): 452-8.

[32] Janssens L, McConnell AK, Pijnenburg M, Claeys K, Goossens N, Lysens R, et al. Inspiratory muscle training affects proprioceptive use and low back pain. Med Sci Sports Exerc. 2015 Jan; 47(1): 12-9.

[33] Kobesova A, Safarova, Marcela RM, Kolar, Pavel. Dynamic neuromuscular stabilization: exercise in developmental positions to achieve spinal stability and functional joint centration. In: Textbook of musculoskeletal medicine [Internet]. Oxford: Oxford University Press; 2016 [cited 2016 Apr 25]. Available from: http://www.tandfonline.com/toc/rwhi20/.

[34] Frank C, Kobesova A, Kolar P. Dynamic neuromuscular stabilization \& sports rehabilitation. Int J Sports Phys Ther. 2013 Feb; 8(1): 62-73.

[35] Kumar S, Sharma VP, Aggarwal A, Shukla R, Dev R. Effect of dynamic muscular stabilization technique on low back pain of different durations. J Back Musculoskelet Rehabil. 2012; 25(2): 73-9.

[36] Kumar S. The spinal column and abdominal pressure changes and their relationship with pain severity in patients with low back pain. Journal of Rheumatology and Medical Rehabilitation. 2009 Jan 1; 13: 1-6.

[37] Hodges PW, Gandevia SC. Changes in intra-abdominal pressure during postural and respiratory activation of the human diaphragm. J Appl Physiol. 2000 Sep; 89(3): 967-76.

[38] van Ramshorst GH, Salih M, Hop WCJ, var Waes OJF, Kleinrensink G-J, Goossens RHM, et al. Noninvasive assessment of intra-abdominal pressure by measurement of abdominal wall tension1. Journal of Surgical Research. 2011 Nov 1; 171(1): 240-4.

[39] Cresswell AG. Responses of intra-abdominal pressure and abdominal muscle activity during dynamic trunk loading in man. Eur J Appl Physiol Occup Physiol. 1993; 66(4): 315-20.

[40] Mesquita Montes A, Gouveia S, Crasto C, de Melo CA, Carvalho $\mathrm{P}$, Santos $\mathrm{R}$, et al. Abdominal muscle activity during breathing in different postural sets in healthy subjects. J Bodyw Mov Ther. 2017 Apr; 21(2): 354-61. 
[41] Ershad N, Kahrizi S, Abadi MF, Zadeh SF. Evaluation of trunk muscle activity in chronic low back pain patients and healthy individuals during holding loads. J Back Musculoskelet Rehabil. 2009; 22(3): 165-72.

[42] Essendrop M, Andersen TB, Schibye B. Increase in spinal stability obtained at levels of intra-abdominal pressure and back muscle activity realistic to work situations. Appl Ergon. 2002 Sep; 33(5): 471-6.

[43] Hagins M, Pietrek M, Sheikhzadeh A, Nordin M. The effects of breath control on maximum force and IAP during a maximum isometric lifting task. Clin Biomech (Bristol, Avon). 2006 Oct; 21(8): 775-80.

[44] Malátová R, Drevikovská P. Testing procedures for abdominal muscles using the muscle dynamometer SD02. Proc Inst Mech Eng H. 2009 Nov; 223(8): 1041-8.

[45] Malátová R, Pucelík J, Rokytovár J, Kolár P. Technical means for objectification of medical treatments in the area of the deep stabilisation spinal system. Neuro Endocrinol Lett. 2008 Feb; 29(1): 125-30.

[46] Malátov R, Rokytová J, Stumbauer J. The use of muscle dynamometer for correction of muscle imbalances in the area of deep stabilising spine system. Proc Inst Mech Eng H. 2013 Aug; 227(8): 896-903.
[47] Urquhart DM, Hodges PW, Story IH. Postural activity of the abdominal muscles varies between regions of these muscles and between body positions. Gait Posture. 2005 Dec; 22(4): 295-301.

[48] Sapsford RR, Hodges PW. Contraction of the pelvic floor muscles during abdominal maneuvers. Arch Phys Med Rehabil. 2001 Aug; 82(8): 1081-8

[49] Hodges PW, Pengel LHM, Herbert RD, Gandevia SC. Measurement of muscle contraction with ultrasound imaging. Muscle Nerve. 2003 Jun; 27(6): 682-92.

[50] Kirkpatrick AW, Brenneman FD, McLean RF, Rapanos T, Boulanger BR. Is clinical examination an accurate indicator of raised intra-abdominal pressure in critically injured patients? Can J Surg. 2000 Jun; 43(3): 207-11.

[51] Triffoni-Melo A de T, Monte-Alegre F de A, Leandro-Merhi VA, Diez-Garcia RW, Triffoni-Melo A de T, Monte-Alegre F de A, et al. Additional abdominal measurements are a useful tool to evaluate body composition in obese women. Arquivos de Gastroenterologia. 2019 Sep; 56(3): 294-9. 